\title{
L'ivresse en Mésopotamie : de la plénitude des sens à la déraison
}

Drunkenness in Ancient Mesopotamia: from Sensorial Satisfaction to Folly

\section{Anne-Caroline Rendu Loisel}

\section{(2) OpenEdition}

1 Journals

Édition électronique

URL : http://journals.openedition.org/mythos/583

DOI : $10.4000 /$ mythos.583

ISSN : 2037-7746

Éditeur

Salvatore Sciascia Editore

\section{Édition imprimée}

Date de publication : 1 décembre 2017

Pagination : 37-47

ISSN : $1972-2516$

\section{Référence électronique}

Anne-Caroline Rendu Loisel, «L'ivresse en Mésopotamie : de la plénitude des sens à la déraison », Mythos [En ligne], 11 | 2017, mis en ligne le 24 septembre 2019, consulté le 28 septembre 2019. URL: http://journals.openedition.org/mythos/583 ; DOI : 10.4000/mythos.583 


\section{L'ivresse en Mésopotamie : de la plénitude des sens à la déraison}

\section{Anne-Caroline Rendu Loisel}

\section{Résumé}

Les textes littéraires rédigés en sumérien au début du 2 millénaire av. n. è., rapportent des scènes de banquet où le moteur semble être la consommation d'alcool - bière ou vin - en grande quantité. Moment de festivité communautaire, le banquet est aussi un espace caractérisé par les optima sensibles que ce soit sur le plan gustatif, auditif, visuel ou tactile. Les effets physiques de l'ivresse se font ressentir chez tous les convives qui s'abandonnent à cet état de plénitude. La poésie amoureuse sumérienne associe l'ivresse au sentiment amoureux. Au-delà du plaisir des sens, il est aussi un événement important pour un groupe d'individus qui sanctionnent de grandes décisions. Mais ce débordement d'alcool peut aussi être dangereux pour l'équilibre des puissances divines.

\begin{abstract}
In the literature of ancient Mesopotamia, especially in Sumerian texts of the beginning of the 2 nd millennium BC, the banquet seems to play an important role. There, alcoholic beverages, beer or wine, are drunken in abundance. In this particular community event, the place is characterized by sensory optima with positive gustatory, auditory, visual and tactile effects. All of the participants feel in their body a new physical and emotional state, a kind of plenitude, in which they abandon themselves. Sumerian love poetry frequently associates the state of being drunk to the one of being in love. But drinking too much may also lead to mythological dangerous situations for the balance of the divine powers.
\end{abstract}

\section{Mots clefs}

Mésopotamie - sumérien - alcool • sensation - banquet

\section{Keywords}

Mesopotamia - Sumerian - alcohol • sensation - banquet

Gilgameš, où cours-tu ? La vie que tu poursuis tu ne la trouveras pas. Lorsque les dieux ont créé l'humanité, c'est la mort qu'ils ont réservé à l'humanité ; ils ont retenu la vie dans leurs mains. Toi, Gilgameš, que ton ventre soit repu, jour et nuit réjouis-toi, chaque jour fais la fête, jour et nuit danse et joue de la musique ; que tes vêtements soient propres, ta tête bien lavée, lave-toi à (grande) eau ; contemple le petit qui te tient par la main, que la bien-aimée se réjouisse en ton sein ! C'est cela le destin de l'humanité !'.

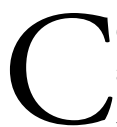

ette célèbre citation de l'épopée ninivite de Gilgameš résume assez bien la réflexion des anciens Babyloniens sur leur relation avec les entités divines : lorsque les dieux ont créé l'humanité, ils se sont gardé l'immortalité. Il ne reste alors au genre humain qu'à profiter de son temps ici-bas par des joies que l'on pourrait dire "simples » : être auprès des gens

$1 \quad{ }^{\mathrm{d}} \mathrm{GIŠ}$ e-eš ta-da-a-al / ba-la-țam ${ }_{2} \check{s} a$ ta-sa-ah-hu-ru la tu-ut-ta / i-nu-ma DINGIR.MEŠ ib-nu-u $a$-wi-lu-tam / mu-tam iš-ku-nu a-na a-wi-lu-tim / ba-la-țam ${ }_{2}$-na qa -ti-šu-nu iṣ-ṣa-ab-tu / at-ta dGIŠ lu ma-li ka-ra-aš-

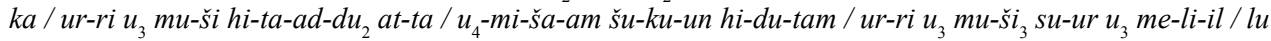
ub-bu-bu șu $u_{2}-b a !(\mathrm{KU})-t u$-ka / qa $a_{2}-q a_{2}$-ad-ka lu me-si me-e lu ra-am-ka-ta / șu-ub-bi șe-eh-ra-am șa-bi-tu

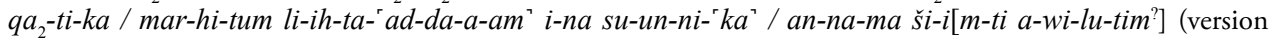
paléo-babylonienne, fragment Meissner, iii 1.1'-14' ; trad. ACRL ; cf. George 2003, 278 pl. 19). Pour le présent article, les références aux sources antiques suivent les abréviations du Chicago Assyrian Dictionary ; les textes en sumérien sont transcrits à partir des textes composites établis sur le site d'eTCSL : http://etcsl.orinst.ox.ac.uk/. 
que l'on aime, au cœur d'une société qui se distingue du sauvage par ses parures, ses parfums, et surtout ses fêtes, un leitmotiv que l'on retrouve dans d'autres textes littéraires akkadiens.

Qu'il soit familial, diplomatique ou rituel, le banquet est une composante essentielle de la vie sociale au Proche-Orient ancien. Maria-Grazia Masetti Rouault souligne très justement que " les scènes de banquet semblent être employées surtout pour exprimer la mise en place de rapport équilibrés et équitables, dans une relation sociale, entre partenaires différents. La présence et l'échange de nourriture, et surtout de boissons alcoolisées, loin de représenter l'excès et le luxe, servent plutôt de révélateurs de la réalité de ces liens et de ces rapports, ou bien servent à souligner leur actualité et leur réussite $"^{2}$. Le Pauvre Homme de Nippur, un texte satyrique du $1^{\text {er }}$ millénaire av. $n$. è., décrit au départ la misère du protagoniste : il n'a qu'un seul vêtement qu'il ne peut pas changer et surtout, il ne rêve que de banqueter. Au-delà de la simple nourriture, c'est aussi le souhait de renouer avec la société et son rang social disparu. Le banquet est un lieu d'abondance de mets de toutes sortes et de boissons, surtout la bière (kaš en sumérien, šikaru en akkadien) et dans un second temps le vin (geštin en sumérien, karānu en akkadien). On trouve préférentiellement différentes variétés de bière, dont les techniques de production semblent avoir été maîtrisées très tôt dès le néolithique ${ }^{3}$. L'akkadien qerìtu désigne les banquets, tant ceux organisés par les hommes que ceux qu'on offrait aux dieux. Son pendant sumérien kaš de 2 -a témoigne de la place prédominante de la bière, puisqu'il signifie, littéralement, " la bière versée ». Ainsi la consommation de cette boisson, peut-être en excès, définissait le principe même du banquet et on peut même aller jusqu'à dire qu'elle en était le moteur ${ }^{4}$.

L'abus de bière, comme de toute boisson alcoolisée, amène l'ivresse et l'expression " dans sa bière " signifie en fait " agir en état d'ivresse " (expression attestée dans des textes paléo-assyriens du début du $2^{\mathrm{e}}$ millénaire av. n. è. $)^{5}$. Dans la Malédiction d'Akkad, écrit en sumérien et relatant la chute de la cité du même nom au cours du $22^{\mathrm{e}}$ siècle av. n. è., les effets physiques de l'ivresse - perte de contrôle de soi et de l'équilibre - servent d'image à l'effondrement de la ville : «que tes esprits gardiens à ton portail soient jetés au sol tels des jeunes hommes vigoureux ivres sous l'effet du vin $»^{6}$. On retrouve quelques témoignages relatifs à une consommation déraisonnée d'alcool dans les textes médicaux $^{7}$ : sans surprise, il y est question de troubles de la perception visuelle, de perte de l'équilibre du corps et de problèmes l'élocution :

Si une personne boit de la bière fine/de qualité et qu'ensuite elle est instable sur ses jambes et que sa vue diminue, pour la guérir...8.

Si une personne boit de la bière fine/de qualité, et que sa tête continue de la faire souffrir,

2 Masetti-Rouault 2004, 50.

3 BRENIQUeT 2009. La bière semble avoir été si importante pour la Mésopotamie que certains chercheurs - par plaisanterie - ont suggéré que c'est pour produire de la bière que les communautés humaines ont développé l'agriculture ! Pour une introduction au vin et à la bière au Proche-Orient ancien, on se reportera à l'ouvrage collectif édité par Lucio Milano en 1994, intitulé Drinking in Ancient Societies, History and Culture of Drinks in the Ancient Near East, Padoue : Milano 1994. On consultera aussi Powell 1995, Glassner 1991, et plus récemment FaIVre 2013 et Lion 2013.

4 Вотте́Ro 1994, 4.

5 Michel 2009.

6 dub-la ${ }_{2}$ la-ha-ma sug ${ }_{2}$-sug ${ }_{2}$-ga-bi / ĝuruš mah ĝeštin naĝ-a-gin ${ }_{7}$ ki-še ${ }_{3}$ he ${ }_{2}$-em-ta-ĝa -ĝa $_{2}$ (Malédiction d'Akkad, 1. 229-230; eTCSL 2.1.5).

7 SCURlock, ANDERSEN 2005, 382.

8 DIŠ NA KAŠ.SAG NAG-ma SUHUŠ.MEŠ-š $u_{2} p a-a l$-qa di-ig-la ma-a-ți ana TI-šu $u_{2} . .$. (BAM 575 iii 49 ). 
qu'elle oublie constamment ses mots, son esprit est confus (lit. 'n'est pas saisi'), ses yeux son immobiles, pour la guérir...?.

Si ses mots sont étranges, et qu'il réclame du vin à intervalles réguliers (alors qu'il a été malade depuis 10 jours... $)^{10}$.

Mais il s'agit ici à chaque fois d'une expérience solitaire de l'ivresse qui differe de celle proprement sociale et collective qu'est le banquet : dans les textes de la littérature proche-orientale ancienne, le banquet est moins une beuverie qu'une nécessaire rencontre joyeuse et festive d'une communauté. On boit ensemble ; les effets peuvent être partagés permettant alors de vivre un moment de plénitude sensorielle, ou au contraire, les méfaits peuvent être ressentis chez un seul individu qui perd tout usage de la raison et de sa conscience. L'autre ou les autres peuvent alors en jouer et en profiter ou au contraire. Le banquet devient un temps liminaire, exceptionnel où la réalité et l'équilibre des puissances peuvent être modifiés pour le meilleur et pour le pire ${ }^{11}$. Les textes mythologiques et hymniques rédigés en sumérien au début de l'époque paléo-babylonienne ( $1^{\text {ère }}$ moitié du $2^{\mathrm{e}}$ millénaire av. notre ère) rapportent plusieurs scènes où les débordements d'alcool ont des conséquences - parfois dramatiques - pour l'équilibre des pouvoirs divins ou les rapports entre individus ${ }^{12}$. Les poèmes décrivant l'amour qui unit la déesse Inana et le dieu Dumuzi ou les hymnes à Ninkasi la déesse sumérienne de la bière illustrent comment l'ivresse peut conduire à une plénitude de l'expérience sensible, multi-sensorielle et partagée. Les textes mythologiques présentent quant à eux, une ivresse divine souvent manipulée par un dieu en vue d'obtenir le consentement, l'approbation de la communauté ou de profiter de l'état de faiblesse pour mieux s'emparer des pouvoirs. Une seule ivresse, mais deux conséquences opposées ${ }^{13}$.

\section{L'ivresse et la plénitude des sens}

Les textes de la littérature cunéiforme le montrent bien : si la bière est fondamentale pour un banquet, d'autres stimulations sont aussi mises en jeu. Les tables sont remplies de mets fins et sucrés, comme les fruits, les gâteaux ; les meilleures pièces de viandes sont apportées et présentées dans de la vaisselle brillante en matières précieuses. La musique, que ce soit par les chants ou les instruments de musique, contribue à créer un cadre sonore agréable auquel s'ajoutent d'autres sons très évocateurs. L'hymne adressé à Inana par le roi d'Isin Iddin-Dagan (Iddin-Dagan A, $\mathrm{XX}^{\mathrm{e}}$ siècle av. n. è. $\left.{ }^{14}\right)$ célèbre l'union de la déesse avec le roi au cours d'une fête

9 DIŠ NA KAŠ.SAG-ma SAG.DU-su DIB.DIB-su INIM.MEŠ- $\check{s} u_{2}$ im-ta-na-aš $\check{S}_{2}-s ̌ i$ ina DUG $_{4} \cdot \mathrm{DUG}_{4}-\check{s u}_{2} u_{2}-p a-$

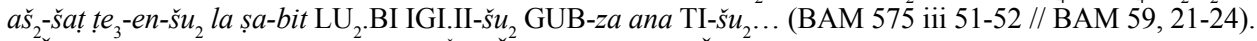

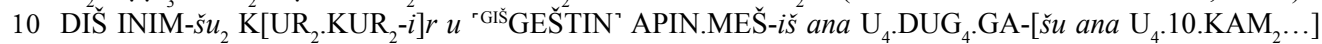
(DPS VII B r. 14 = TDP 66, 1.70').

11 Masetti-Rounalt 2004, 62.

12 Pour une présentation analytique de la littérature sumérienne, on se reportera à VeLDHUIs 2003.

13 Les données littéraires mésopotamiennes ne permettent pas, à notre connaissance, d'aborder la question de l'ivresse ritualisée ou de toute autre modification des états de conscience dans le cadre de rituel, à l'instar de ce qui se passe pour les chamanes dans les Andes boliviennes (voir notamment l'article de GeFfroy 2017, où l'ivresse est un moyen pour le chamane d'accéder au monde de l'invisible). Les pistes sont peut-être à chercher également du côté de l'archéologie et de l'iconographie : pour ces questions, on se reportera aux travaux de Diana Stein par exemple sur la possible ingestion de substances (STEIN 2017).

14 On a à notre disposition quatorze tablettes et fragments permettant de reconstituer ce texte de 230 lignes environ. Iddin-Dagan est un roi de la $1^{\text {ère }}$ Dynastie d'Isin, ville du Sud de la Mésopotamie, et aurait régné entre 1974 et 1954 av. n. è.. Bibliographie sélective : JaCOBSen 1987 et JaCobSen 1997 ; Reisman 1973 ; Kramer 1983. 
qui implique tous les membres de la communauté. La liesse collective est à son comble dans un débordement d'optima sensoriels : matières chatoyantes, gâteaux de miel et fruits alléchants... même le son produit par les bulles de bières dans les récipients font de ce banquet rituel un temps hors de la vie quotidienne où monde divin et humain se mêlent :

Dans les sanctuaires de l'humanité, de l'encens à entasser est approché d'elle comme une forêt odorante de cèdres. On y sacrifie des moutons alum, des moutons à longue laine et des moutons engraissés. Pour la nugig (Inana), on purifie le sol, on accomplit pour elle les lustrations. On célèbre cela dans un chant ! Beurre, dattes, fromages et toutes sortes de fruits. Les prémices (ce qu'il y a de meilleur) débordent pour elle dans les tables du pays. On lui verse de la bière brune, on lui verse de la bière blonde, la bière brune, la bièreépeautre/amidonnier, pour ma Dame, la bière-épeautre/amidonnier, les récipients šaggub et lamdir ont glouglouté dubul dabal za (lit. 'ont fait blop-blop') à cause d'elle ${ }^{15}$.

L'expression dubul dabal za est construite avec le verbalisateur za « faire le bruit $-\mathrm{x}$ » accompagné du terme dubul dabal à forme onomatopéique caractéristique ${ }^{16}$ reproduisant le son des bulles de fermentation dans les containers en argile. Ce banquet rituel devient alors un espace et un moment de saturation sensorielle positive, et une expérience fondamentalement communautaire. Les enjeux peuvent y être importants.

Dans le récit en sumérien que l'on appelle communément Le voyage d'Enki à Nippur, le grand dieu Enki vient de construire sa demeure terrestre à Eridu dans le sud de la Mésopotamie. C'est un récit de 130 lignes environ qui a été mis par écrit vers la fin du $3^{\mathrm{e}}$ millénaire av. n. è. Enki/Éa (Enki étant son nom sumérien et Éa son nom akkadien) est le grand dieu de la sagesse, des savoirs et savoir-faire. Il est aussi le dieu des eaux douces et des eaux souterraines, cette nappe aquatique nommée l'Abzu/Apsû qui court sous la surface de la terre. Son temple principal était bâti à Eridu (l'actuelle Abu Sahrain), située sur la rive droite de l'Euphrate, dans les marais du sud de la Mésopotamie. Il s'agit de la plus méridionale et une des plus anciennes des grandes cités sumériennes. Le temple que le dieu se fait construire est un espace qui mêle les stimulations sensorielles : le bâtiment est en lapis-lazuli et en or, il "mugit " nous dit le texte ; les vergers sont remplis de fruits murs et alléchants, tandis que les petits poissons frétillent dans les eaux. Une fois son temple construit, Enki se rend à Nippur, autre cité sumérienne, ville du grand dieu Enlil. Enki se rend auprès de son père et lui apporte tout ce qui est nécessaire à une fête :

Enki avait (en partant) mis à mort taureaux et moutons en abondance. Il avait fait venir les tambours-alû qui n'étaient pas présents, les tambours-ub zabar là où il n’y en avaient pas. Ayant pris le chemin de Nippur, il introduisit avec lui tout cela dans le gigunû, la pure chapelle de Nippur. Enki fit apporter le vin, fit apporter la bière. Il versa le vin dans/par de larges cratères-zabar. Il prépara la bière d'épeautre. Dans le kurkuru, il mélangea le moût (bouillie de malt) de la très bonne bière. Il versa jusqu'au bord du sirop de dattes en quantité égale. Il brassa ensemble cette bouillie douce pour qu'elle

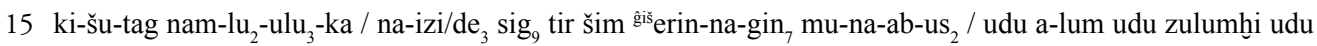
niga ĝiš-- mu-ni-ib-tag-ge / nu- $\mathrm{u}_{8}$-gig-ra ki mu-na-an-sikil-e šir ${ }_{3}$-ra mu-ra-an-gal $\mathrm{I}_{2} / \mathrm{i}_{3}$-nun $\mathrm{zu}_{2}$-lum ga-ar niğ $\hat{g}_{2}$-sa-ḩa 7-bi / gišsbanšur kalam-ma-ka nesaĝ mu-na-ab-si / kurun gig mu-na-ra-de ${ }_{2}(-\mathrm{e})$ / kurun babbar

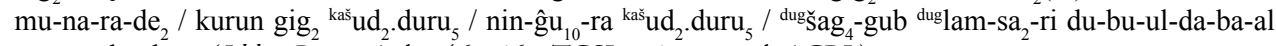
mu-un-da-ab-za (Iddin-Dagan A, 1. 146-156, eTCSL 2.5.3.1, trad. ACRL).

16 Pour les formes onomatopéiques en sumérien, on se reportera à BLACK 2003. 
rafraîchisse. Enki qui était dans le sanctuaire de Nippur offrit du pain à son père Enlil (...) Tous buvaient la bière et profitaient du vin. Ils remplirent les vases-aga, et dans le ciel et sur la terre, ils rivalisaient par (les récipients en) bronze» ${ }^{17}$.

Enki installe les convives ; tout ce beau monde ne fait pas que de boire, et le banquet devient une expérience poly-sensorielle, savamment préparée par Enki : il y a de la nourriture avec les animaux sacrifiés mentionnés au début, et de la musique pour se distraire ; mais le banquet est principalement représenté par cette abondance d'alcool, où se mêlent le vin et la bière qu'Enki lui-même a préparés sur place. Les effets escomptés ne se font pas attendre. En réunissant ainsi l'assemblée des dieux, Enki cherche leur approbation quant à la construction de sa demeure d'Eridu. Sous les effets de l'alcool, ils sont en joie, et le grand dieu lui-même, le père Enlil donne officiellement son accord, dans un parallèle avec l'usage du banquet sur terre qui vient ratifier une alliance :

Une fois la bière bue et le vin savouré, et une fois... Enlil était tout joyeux dans son palais de Nippur. Enlil déclara alors aux Annuna : 'Grands dieux qui vous tenez présents ici, Anunna qui êtes alignés dans l'assemblée (des dieux), mon fils, le roi Enki, s’est construit une demeure !18

Mais la vraie motivation du banquet n'est-elle pas d'obtenir cette approbation communautaire ? Le banquet apparaît comme une expérience poly-sensorielle d'émulation collective, dans une forme de manipulation par une substance qui met en joie, dans un contexte de débordements d'effets sensoriels plaisants. Cette combinaison d'optima sensorielle se retrouve également dans la poésie amoureuse sumérienne, contemporaine des textes précédemment évoqués. Le vin et la bière font partie de ces substances que l'on pourrait qualifier d'aphrodisiaques, qui participent à la représentation métaphorique de l'expérience sensuelle de l'être aimé, de son corps et de son être. Dans un dialogue entre la déesse Inana et son amant Dumuzi ${ }^{19}$, le vin est cité aux côtés du miel :

Mon amour, mon amour, mon amour,

ma chérie, ma chérie, mon miel de la mère qui l'a engendrée,

Mon vin sirupeux, ma douceur de miel, ma bouche melliflue de sa mère.

Tes yeux - leur regard me réjouit, viens ma sœur bien-aimée !

Ta bouche - sa parole me réjouit, ma bouche melliflue de sa mère !

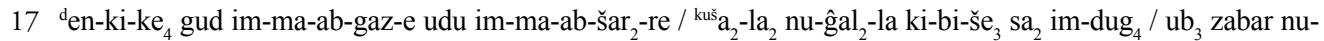

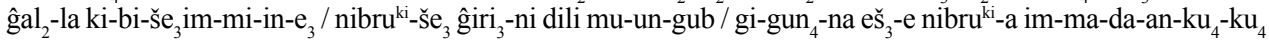
$/$ den-ki-ke ${ }_{4}$ kaš-kaš-e ba-tekurun ${ }_{2}$-kurun ${ }_{2}$-e ba-te / kurun ${ }_{2}$ gal zabar-ra ba-ni-in-de ${ }_{2} /$ kašulušin dili-am ${ }_{3}$ ba-ni-in-

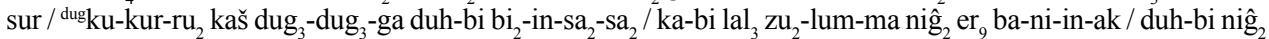

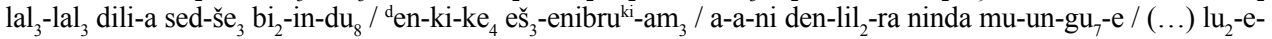
ne kaš $\mathrm{i}_{3}$-na ${ }_{8}$-na ${ }_{8}$-ne kurun im-dug ${ }_{3}$-ge-ne / zabaraga im-gur ${ }_{4}$-gur 4 -re-e-ne zabar-e an uraš-e a-da-min 3 mu-un-dine (Le voyage d'Enki à Nippur, 1. 93-105; 1. 111-112. Transcription eTCSL 1.1.4, trad. ACRL). Pour l'expression " ils rivalisaient par (les récipients en) bronze ", c'est-à-dire : " c'est à qui boira le plus ", en suivant la proposition d'Attinger 1993, p. 127, et avec la note 100 relative à l'expression " au ciel et à la terre » : « ici, peut-être expression ampoulée pour «partout » v.s. recherche d'un effet comique dans la description de l'incroyable beuverie ».

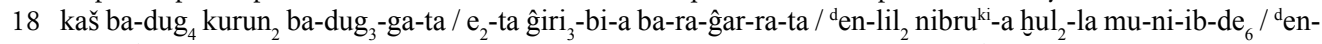
lil ${ }_{2}-$ le da-nun-na-ke $_{4}$-ne gu ${ }_{3}$ mu-na-de ${ }_{2}$-e / diĝir gal-gal-la $\mathrm{i}_{3}$-sug ${ }_{2}$-ge-en-za-na / da-nun-na ub-šu-unken-na-ka si mu-un-sa ${ }_{2}-\mathrm{sa}_{2}$-e-en-za-na / dumu-ĝu ${ }_{10} \mathrm{e}_{2}$ mu-un-du lugal den-ki-ke $_{4}$ (Le voyage d'Enki à Nippur, l. 114-120 ; transcription eTCSL 1.1.4, trad. ACRL).

19 Pour ces textes, voir Sefati 1998. 
Tes lèvres - leur baiser me réjouit, viens ma sœur bien-aimée !

Ma sœur, ton orge - sa bière est délicieuse, ma bouche melliflue de sa mère !

Ton pain de bière - sa bière est exquise, viens ma sœur bien-aimée !

Dans la maison - tes charmes sont irrésistibles, ma bouche melliflue de sa mère !20

Bière et miel sont deux substances pluri-sensorielles : elles évoquent non seulement une stimulation gustative plaisante - parce que sucrée et réjouissante - mais aussi visuelle tout autant agréable : les deux liquides de couleur similaire ne sont pas sans évoquer l'or, sa luminosité et sa brillance. Le miel est en outre fréquemment associé dans la littérature suméro-akkadienne aux paroles divines, aussi douce à entendre que le miel sur la langue ${ }^{21}$. Leur association en contexte métaphorique n'est probablement pas à éloigner du fait que le miel est employé dans le processus de fabrication de la bière.

Une chanson à boire en l'honneur de la déesse-bière Ninkasi, du début du $2^{\mathrm{e}}$ millénaire av. n. è., décrit ces effets physiques plaisants provoqués par la consommation de cette substance euphorisante qui est évoquée au début du passage non par son goût mais par le bruit produit lorsqu'on verse la bière :

Que Ninkasi soit à tes côtés! Qu'elle verse de la bière et du vin pour toi ! Que (le versement de) la douce liqueur résonne noblement pour toi! La douce bière est dans les containers d'herbes-bur. Je ferai se tenir les échansons, les garçons et les brasseurs. Tandis que je tourne autour du lac de bière, en me sentant bien, en me sentant bien (=gur 4 littéralement " être épais "), en buvant de la bière dans un état de bien-être, en buvant du vin et étant dans l'allégresse, la joie au cœur, mon intérieur joyeux : mon cœur est un cœur rempli de joie ! J'habille mon intérieur satisfait d'un manteau d'apparat-pala ${ }^{22}$

Sur la tablette ${ }^{23}$ qui contient le texte d'où est tire le passage précédent, on trouve également un autre hymne à la déesse bière Ninkasi (Ninkasi A). Au cours de cet hymne, on précise que la déesse place le vase à fermentation " qui fait un son noble " $\left(\mathrm{gu}_{3}\right.$ nun di-dam, 1. 43). Les textes de la littérature suméro-akkadienne nous donnent également l'image de la taverne comme un lieu de débauche et en fait une maison de la prostitution. La déesse Bau décrit les parties génitales de la tavernière comme étant aussi douce et agréable que la bière qu'elle sert :

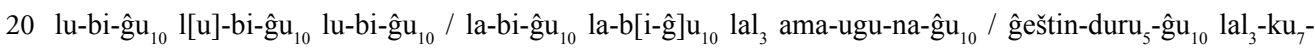

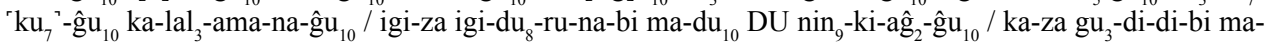

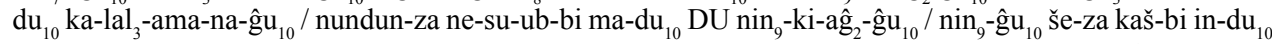

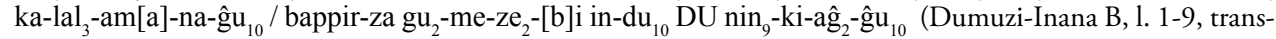
cription Sefati 1998, 128, trad. ACRL).

21 "Que la déclaration de ses lèvres (Marduk) soit aussi douce que le miel pour l'humanité " (PInches, Texts in

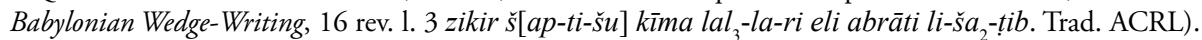

22 dnin-ka-si za-da hu-mu-u ${ }_{8}$-da-an-til ${ }_{3} /$ kaš g geštin hu-mu-ra-an-bal-bal-e / kurun ${ }_{2}$ lal $_{3}$-e gu ${ }_{3}$ nun ha-ra-ni-ib-be ${ }_{2}$ / gibuni $\hat{g}(\mathrm{SUG}){ }_{2}^{\mathrm{u}} \mathrm{bur}_{2}$-a kaš $\mathrm{ku}_{7}-\mathrm{ku}_{7}$-dam / sagi lu $\mathrm{d}_{2}$-tur-ra lunga $\mathrm{bi}_{2}$-in-gub-en / a-niĝin ${ }_{2}$-e niĝin-na-ĝ $\mathrm{u}_{10}$-ne

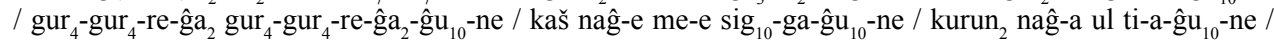

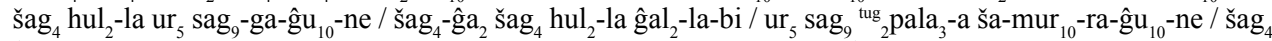
dinana ki-bi ba-ab-gi ${ }_{4} / \breve{s ̆ a g}_{4}$ ga-ša-an-an-na-ke ${ }_{4}$ ki-bi ba-ab-gi ${ }_{4} /[\ldots]$ [ ${ }^{\mathrm{d}}$ nin]-ka-si-kam (A Drinking Song, 1. 18-29, transcription eTCSL 4.23.1, trad. ACRL). Voir l'édition de Civil 1964.

23 Trois exemplaires sont attestés : AO 5385 (TCL 15 20) ; Ni 4569 iii 24'ff. (ISET 1 60f.) et VAT 6705 (VAS 10 156). Ils permettent de reconstruire les deux textes présentés ici, à savoir l'hymne à Ninkasi et la chanson à boire, les deux textes étant conservés sur les mêmes tablettes. 
... la bière de la Cabaretière est douce. Comme sa bière, son sexe est doux, sa bière est douce. Comme sa bouche, son sexe est doux, sa bière est douce. Sa bière diluée, sa bière est douce. Mon Šu-Sīn qui m’a réjouie, qui m’a réjouie, qui m’a ravie, mon Šu-Sin qui m’a réjouie, mon $\check{S}_{u}$-Sĩn, bien-aimé d'Enlil, mon roi, le dieu de son pays ! ${ }^{24}$

Combinés aux autres effets sensoriels positifs créés comme la musique, les matières précieuses, les mets, etc. les effets de l'alcool amènent à une nouvelle compréhension du monde, de son organisation et des rapports qu'on entretient avec l'autre. Cette nouvelle expérience sensible, profondément pluri-sensorielle, ne peut être bénéfique qu'à condition d'être partagée. Trop de stimulations agréables dérègleraient aussi le mode de pensée, faisant tomber la méfiance et le raisonnement.

\section{L'ivresse et la déraison}

Dans le texte intitulé les Instructions de Šuruppak (rédigé en sumérien et remontant au début du $2^{\mathrm{e}}$ millénaire av. n. è.), un roi met en garde son fils Ziusudra ${ }^{25}$ contre de funestes comportements. Parmi ceux-ci, on trouve celui de choisir une épouse en pleine fête collective : le cadre festif avec l'éclat des matières précieuses participent à la tromperie générale. Tout n'est qu'illusion et on ne peut avoir un jugement clair $^{26}$.

Le banquet semble être l'occasion pour le grand dieu Enki d'obtenir ce qu'il souhaite, dans le texte sumérien mentionné plus haut. Mais ceci peut avoir des conséquences dramatiques si la personne initiatrice est animée de mauvaises intentions. C'est ce qu'Enki expérimente contre son gré dans Inana et Enki: Inana (Ištar en akkadien), déesse de la fécondité, particulièrement jalouse et vindicative, se rend auprès d'Enki. Elle est accueillie en grande pompe par un banquet:

Lorsqu'Inana sera entrée dans l'Apsû d'Eridu, donne-lui à manger des gâteaux au beurre, et qu'on lui verse de l'eau fraîche, régal du cœur ! Devant le Lion, fais-lui boire de la bière $!^{27}$

Les deux divinités se retrouvent à la même table et festoient. On a affaire ici à un topos littéraire, qui reprend les mêmes expressions :

Enki et Inana, côte à côte, dans l'Abzu, buvaient la bière et appréciaient le vin. Ils

24 AN X X X-ĝn ${ }_{10}$ za-bi-tum-ma kaš-a-ni ze -ba-am $_{3} /$ kaš-a-ni-gin ${ }_{7}$ gal $_{4}-$ la-ni ze $_{2}$-ba-am 3 kaš-a-ni ze 2 -ba-am 3

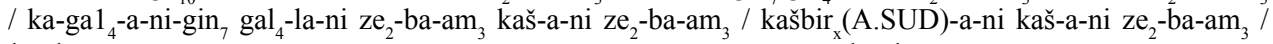

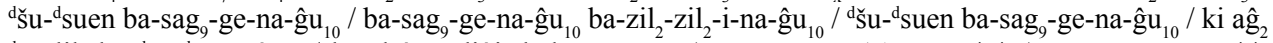

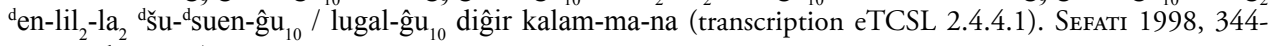
352 , trad. ACRL).

25 Pour le rapprochement entre la cité Šuruppak dans le Sud de la Mésopotamie (mentionné ici comme anthroponyme) et la personnalité de Ziusudra, le Noé mésopotamien, voir l'editio princeps de Alster 1974.

26 ezem-ma-ka dam na-an-tuku-tuku-e / šag ${ }_{4}$-ga huĝ-ĝa $a_{2}-a_{3}$ bar-ra huĝg-ĝa $a_{2}-a_{3} /$ kug huĝ-ĝa ${ }_{2}-a_{3}$ za-gin ${ }_{3}$ huĝ-ĝa $a_{2}-\mathrm{am}_{3} / \operatorname{tug}_{2}{ }^{2}$ huĝ-ĝa $a_{2}-\mathrm{am}_{3}$ gada? huĝ-ĝa ${ }_{2}-\mathrm{am}_{3}$ " Tu ne dois pas choisir une épouse lors d'une fête. Son intérieur est illusion, son extérieur est illusion. L'argent sur elle est emprunté, le lapis-lazuli sur elle est emprunté ; la robe sur elle est empruntée, le vêtement de lin sur elle est empruntée » (Les Instructions de Šuruppak, 1. 208-211, transcription eTCSL 5.6.1; trad. ACRL).

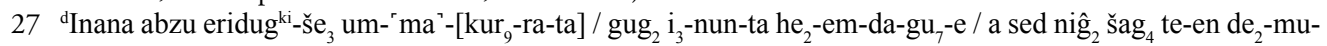
[na]-ni / igi piriôg-ĝa $a_{2}-k^{k}$ kaš hu-mu-[na-na ${ }_{8}$-na $_{8}$ ] (Inana et Enki, segment C 1. 8-11 ; transcription eTCSL 1.3.1, trad. ACRL). 
remplirent les vases-aga à ras-bord, dans le ciel et sur la terre, ils rivalisaient par (les récipients en) bronze (...) À la pure Inana, à mon enfant, je vais donner... ${ }^{28}$

Et Enki donne alors à la déesse tous les pouvoirs divins ; celle-ci les prend et retourne chez elle à Uruk avec. Le réveil d'Enki est dur, une fois dissipés les effets de la bière. Il fait venir son page et lui demande où sont passés tous ces pouvoirs. L'alcool n'excuse pas tout, même chez les dieux ; et lorsqu'Isimud est envoyé chercher Inana, celle-ci accuse Enki de trahison : comment a-t-il pu la tromper de la sorte et revenir sur la promesse faite? Isimud insiste plus de six fois, et la septième fois, Inana semble triompher définitivement. Rapportant les pouvoirs à Uruk, sa ville, la déesse lance une fête collective poly-sensorielle où nourritures, boissons et musique sont répandus en abondance :

... le bateau céleste, la fête.... qu'il récite de grandes prières. Que le roi mette à mort les taureaux et moutons en abondance. Qu'il verse la bière dans un vase-bur. Qu'il fasse retentir les tambours šem et alu, et qu'il fasse jouer le mélodieux tigi. Que les pays étrangers proclament ma majesté, que mon peuple prononce ma prière ${ }^{29}$.

Qu'il y ait une réalité historique derrière cet épisode n'est pas notre propos ici. L'utilisation de l'alcool peut avoir des conséquences dramatiques pour certains, ici la ville d'Eridu. À la fin du texte, Enki n'a d'autres choix que d'abandonner les pouvoirs tout en établissant une alliance étroite entre les deux cités sumériennes.

La mise en danger de l'équilibre des puissances divines par l'alcool se perçoit également dans une autre composition en langue sumérienne, appelée Enki et Ninmah. On la connaît sur des versions paléo-babylonienne mais elle est probablement plus ancienne. Au début du récit, les dieux n'en peuvent plus de travailler. Il faut leur créer un substitut, l'être humain, et c'est Enki qui est à l'origine de cette ingénieuse idée. Pour célébrer cet événement et la sagesse d'Enki, un banquet est organisé dans la communauté divine. Les mets sont apprêtés, la bière coule à flot. Les deux principales divinités qui ont pris part à la création des hommes, c'est-à-dire Enki et la déesse Ninmah, sont d'humeur joyeuse : "ils avaient bu tant de bière que leur cœur enfla (ul te) $»^{30}$. L'ivresse partagée est source de joie ; le verbe composé sumérien employé ul te signifie littéralement " approcher l'allégresse "; il suggère en outre une idée de " gonflement ", d'être " enflé, opulent ». Il a pour équivalent l'akkadien habāṣu " être euphorique, ivre », une manifestation vocale et collective du type chant ou acclamation dans des contextes de fêtes publiques ${ }^{31}$.

Ninmah lance alors un défi à Enki : elle crée des êtres informes et Enki doit leur trouver une utilité. Par exemple, celui dont les mains sont faibles, Enki en fait un fonctionnaire ; celui qui est aveugle, il le transforme en chantre ; la femme stérile, il en fait une courtisane du harem,

28 den-ki dinana e-en-bi-ta / abzu-a kaš im-na ${ }_{8}-n_{8}$-ne kurun im-dug $_{3}$-ge-ne / zabaraga im-gur ${ }_{4}$ gur $_{4}$-e-- ne $^{\urcorner} /$ 'zabar' an uraš-a a-da- $\min _{3}$ mu-un-/akl-[ne] (...)kug d[inana-ra] [dumu-ĝa ${ }_{10}$-ur ${ }_{2}$ ga-na-ab-šum ${ }_{2}$ ba-ra-... (Inana et Enki, Segment C 1. 27-30, segment D 1.1 ; transcription eTCSL 1.3.1, trad. ACRL).

29 x-ta $\mathrm{ma}_{2}$ an-na ezen $\mathrm{x}[\ldots]$ / sizkur $_{2}$ gal-gal hu-mu-un-[...-e] / lugal-e gud he - -em-ma-ab-gaz-e 'udu' [he ${ }_{2}-$

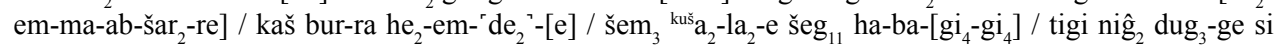

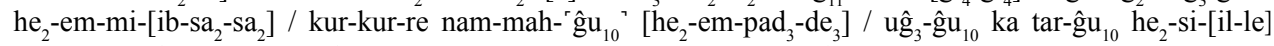
(Inana et Enki, Segment H, 1. 241-248, transcription eTCSL 1.3.1, trad. ACRL).

$30{ }^{\mathrm{d}}$ en-ki-ke ${ }_{4}{ }^{\mathrm{d}}$ nin-mah-e kaš im-na ${ }_{8}-\mathrm{na}_{8}$-ne šag ${ }_{4}$-bi ul mu-un-te (Enki et Ninmah, 1.52. Transcription eTCSL 1.1.2; trad. ACRL).

31 Jaques 2006, 45 et 268. 
etc. En retour, Enki crée un monstre, à qui Ninmah est incapable d'attribuer un destin. Le récit met ainsi en lumière le pouvoir créateur supérieur d'Enki.

On voit surtout dans ces textes que la modification de la conscience d'un dieu peut avoir des répercutions dramatiques sur le cosmos : dans le premier texte, les pouvoirs divins sont confisqués définitivement par une divinité, et dans le deuxième, on assiste à des créations contre-nature ${ }^{32}$. À chaque fois, l'ingéniosité d'un dieu est mise en avant dans une forme de manipulation indirecte de l'autre. C'est en gardant sa propre maîtrise de soi et en exerçant un contrôle sur son corps, qu'Inana ou Enki parviennent à leurs fins. L'excès sensoriel du banquet participe à la privation du sens et de l'exercice de la conscience. L'alcool participe aux franchissements des limites, entraînant des perturbations cosmiques : les êtres créés par Ninmah sont bel et bien présents dans le monde des vivants. L'ivresse apparaît donc moins comme un mode d'action qu'un mode de dérèglement. Elle entraîne un excès d'énergie créatrice qui devient alors mauvaise, car non naturelle, et aux conséquences dramatiques.

Les dieux semblent perdre leurs pouvoirs d'action, ils deviennent dépendants du bon vouloir d'un autre dieu qui a su maîtriser son ivresse. À ma connaissance, il n'existe pas beaucoup de cas où les divinités deviennent faibles, au point de perdre la mémoire, et leurs pouvoirs inactifs. Il y a les moments où un dieu meurt (je pense notamment à Apsû dans l'Enüma eliš : Éa lui a retiré sa couronne, l'a lié et mis à mort), et aussi, les moments où ils sont plongés dans le sommeil. Les dieux ne rêvent pas, mais à leur réveil - tout comme lorsqu'ils dessaoulent - des bouleversements cosmiques ont eu lieu ou sont à prévoir : l'épisode d'Inana qui se fait violer pendant son sommeil par le jardinier Šukaletuda en est l'exemple le plus éloquent.

\section{Conclusion}

Comme le suggérait déjà Piotr Michalowski, le banquet a beaucoup en commun avec le rituel : il est temporaire, il implique une partie spécifique de la communauté, il nécessite une réorganisation des paramètres sensoriels, amenant alors à une nouvelle expérience du monde environnant. De la même façon que dans le rituel, de nouvelles configurations sociales s'expriment, alors qu'elles ne pourraient se faire dans la vie quotidienne. Dans le rituel, monde divin et monde humain se retrouvent ainsi.

Le banquet constitue un espace et un moment social particuliers, qui nécessite un aménagement spécifique de ses paramètres sensoriels : pas de banquet sans musique, chants, éclats de voix, sans mets fins, sucrés, sans luxure, et encore moins sans alcool. La consommation de bière ou de vin va contribuer à une modification de la conscience des individus, et les conséquences peuvent être dramatiques si l'on se trouve dans le monde divin. Il s'agit moins d'un mode d'action, que d'un mode de manipulation indirecte pour l'individu qui parvient à se maîtriser, au détriment de celui qui se laisse aller et perd tout contrôle, de lui-même et de son domaine de compétence.

Anne-Caroline Rendu Loisel Université de Strasbourg Archéologie et histoire ancienne : Méditerranée - Europe (ArcHiMèdE) MISHA - 5 allée du Gal Rouvillois CS 50008 - 67083 Strasbourg Cedex France acrenduloisel@hotmail.com 


\section{Bibliographie}

Alster 1974

B. Alster, The Instructions of Šuruppak, Mesopotamia. Copenhagen Studies in Assyriology 10, Copenhagen 1974.

Attinger I993

P. Attinger, Éléments de Linguistique sumérienne, La construction $d u_{11} / e / d i$ " dire ", FribourgGöttingen 1993.

BLACK 2003

J. Black, "Sumerian Noises: Ideophones in Context ", in Walter Sallaberger, K. Volk, A. Zgoll (eds), Literatur, Politik und Recht in Mesopotamien, Festschrift für Claus Wilcke, Orientalia Biblica et Christina 14, Wiesbaden 2003, 35-52.

\section{BOTTÉRO 1994}

J. Bottéro, "Boisson, banquet et vie sociale en Mésopotamie ", in L. Milano (ed.), Drinking in Ancient Societies, History and Culture of Drinks in the Ancient Near East, Papers of a Symposium held in Rome, May 17-19 1990, History of the Ancient Near East Studies, Padova 1994, 3-4.

Breniquet 2009

C. Breniquet, "Boire de la bière en Mésopotamie... ", in X. Faivre, B. Lion, C. Michel (éds), Et il y eut un esprit dans l'Homme, Jean Bottéro et la Mésopotamie, Maison RenéGinouvès Archéologie et Ethnologie, Paris 2009, 183-196.

Civil 1964

M. Civil, "A Hymn to the Beer Goddess and a Drinking Song ", in R. D. Biggs and J. A. Brinkman (éds), Studies Presented to A. Leo Oppenheim, June 7, 1964, The Oriental Institute of the University of Chicago, Chicago, 1964, 67-89.

\section{FaIVRe 2013}

$\mathrm{X}$. Faivre, «La bière. De la brasserie au cabaret : approche archéologique ", in C. Michel (éd.), L'alimentation dans l'Orient ancien, cuisines et dépendances, Cahier des thèmes transversaux ArScAn volume IX (2011-2012), Nanterre 2013, 374-392.
GefFroy 2017

C. Geffroy, "Boire pour entrer en transe : l'ivresse du chamane dans les Andes boliviennes ", in G. Dumas, M. Fortier, J. C. González (dir.), "Les états modifiés de conscience en question, anciennes limites et nouvelles approches ", Intellectica 67, 1 (2017), 327-346.

George 2003

A. George, The Babylonian Gilgamesh Epic, Introduction, Critical Edition, and Cuneiform Texts, Oxford 2003.

GLASSNER 1991

J.-J. Glassner, « Les dieux et les hommes, le vin et la bière en Mésopotamie ancienne ", in D. Fournier, S. d'Onofrio (éds), Le ferment divin, Paris 1991, 127-146.

\section{JACOBSEN 1987}

T. Jacobsen, That Harps that Once... Sumerian Poetry in Translation, Yale 1987.

JaCOBSEN 1997

T. Jacobsen, " Sumerian Canonical Compositions. B. Royal Focus 2. Royal Hymns : The Sacred Marriage Compositions from the Biblical World ", in W. W. Hallo (ed.), The Context of Scripture I : Canonical Compositions from the Biblical World, Brill, Leiden 1997, 554-559.

JAQUeS 2006

M. Jaques, Le vocabulaire des sentiments dans les textes sumériens. Recherche sur le lexique sumérien et akkadien, Alter Orient und Altes Testament 332, Münster 2006.

\section{Kramer 1983}

S. N. Kramer, Le mariage sacré, Paris, 1983.

LION 2013

B. Lion, "Les cabarets à l'époque paléobabylonienne ", in C. Michel (éd.), L'alimentation dans l'Orient ancien, cuisines et dépendances, Cahier des thèmes transversaux ArScAn volume IX (2011-2012), Nanterre 2013, 393-400.

Masetti-Rouault 2004

M.-G. Masetti-Rouault, « Les dangers du 
banquet en Mésopotamie ", in M. Mazoyer, J. Pérez Rey, F. Malbran-Labat, R. Lebrun (éds), La fête. La rencontre des dieux et des hommes, KUBABA, Paris 2004, 49-66.

Michalowski 1994

P. Michalowski, «The Drinking Gods : Alcohol in Mesopotamian Ritual and Mythology ", in L. Milano (ed.), Drinking in Ancient Societies, History and Culture of Drinks in the Ancient Near East, Papers of a Symposium held in Rome, May 17-19 1990, History of the Ancient Near East Studies, Padova 1994, 27-44.

Michel 2009

C. Michel, "'Dis-moi ce que tu bois...' Boissons et buveurs en Haute Mésopotamie et en Anatolie au début du IIe millénaire av. J.C. ", in X. Faivre, B. Lion, C. Michel (éds), Et il y eut un esprit dans l'Homme, Jean Bottéro et la Mésopotamie, Maison René-Ginouvès Archéologie et Ethnologie, Paris 2009, 197220.

\section{Milano 1994}

L. Milano "Vino e birra in Oriente. Confini geografici e confine culturali ", in L. Milano (ed.), Drinking in Ancient Societies, History and Culture of Drinks in the Ancient Near East, Padova 1994, 421-440.

Milano 1994

L. Milano (ed.), Drinking in Ancient Societies, History and Culture of Drinks in the Ancient Near East, Padova 1994.

\section{POWELl 1995}

M. A. Powell, "Wine and Vine in Ancient Mesopotamia : the Cuneiform Evidence ", in P. E. McGovern, S. J. Fleming, S. H. Katz (eds), The Origins and Ancient History of Wine, Philadelphia, 97-122.

ReISMAN 1973

D. D. Reisman, " Iddin-Dagan's Sacred Mariage Hymn ", Journal of Cuneiform Studies 25 (1973), 185-262.

\section{Sefati 1998}

Y. Sefati, Love Songs in Sumerian Literature, Jerusalem, 1998.

Scurlock, Andersen 2005

J. Scurlock, B. A. Andersen, Diagnoses in Assyrian and Babylonian Medicine, Ancient Sources, Translations, and Modern Medical Analyses, Urbana-Chicago 2005.

STEIN 2017

D. Stein, "The Role of Stimulants in Early Near Eastern Society: Insights through Artifacts and Texts ", in Y. Heffron, A. Stone, M. Worthington (eds), At the Dawn of History, Ancient Near Eastern Studies in Honor of J. N. Postgate volume 1, Winona Lake 2017, $507-$ 533.

Veldhuis 2003

N. Veldhuis, "Sumerian Literature », in G. J. Dorleijn, H. L. J. Vanstiphout (eds), Cultural Repertoires: Structure, Function and Dynamics, Leuven 2003, 29-44. 